CARDIOLOGY

\title{
Floppy Mitral Valve (FMV)/Mitral Valve Prolapse (MVP) and the FMV/MVP Syndrome: Pathophysiologic Mechanisms and Pathogenesis of Symptoms
}

\author{
Konstantinos Dean Boudoulas ${ }^{a, b}$ Harisios Boudoulas ${ }^{b, c, d}$ \\ a Division of Cardiovascular Medicine, Section of Intervention Cardiology, at ${ }^{\mathrm{b}}$ The Ohio State University,

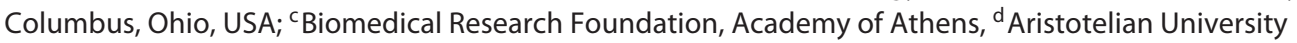 \\ of Thessaloniki, Thessaloniki, Greece
}

\section{Key Words}

Floppy mitral valve · Mitral valve prolapse $\cdot$ Mitral valve regurgitation · Floppy mitral valve/mitral valve prolapse syndrome

\begin{abstract}
Mitral valve prolapse (MVP) results from the systolic movement of a portion or segments of the mitral valve leaflets into the left atrium during left ventricular systole. It is well appreciated today that floppy mitral valve (FMV) is the central issue in the MVP and mitral valve regurgitation (MVR) story. The term FMV refers to the expansion of the area of the mitral valve leaflets with elongated chordae tendineae, chordae rupture and mitral annular dilation. FMV/MVP occurs in a heterogeneous group of patients with a wide spectrum of mitral valve involvement from mild to severe. Two types of symptoms can be defined in FMV/MVP patients. In one group of patients, symptoms are directly related to progressive MVR. In the other group, symptoms cannot be explained by the degree of MVR alone; activation of the autonomic nervous system has been implicated for the explanation of symptoms in this group of patients which is referred to as the FMV/MVP syndrome. In this brief review, the natural history, pathophysiologic mechanisms and management of patients with FMV/MVP/MVR and FMV/MVP syndrome are discussed.

(c) 2013 S. Karger AG, Basel
\end{abstract}

(C) 2013 S. Karger AG, Base

0008-6312/13/1262-0069 \$38.00/0

\section{Introduction/Definitions}

Mitral valve prolapse (MVP) results from the systolic movement of portion(s) or segments of the mitral valve leaflets into the left atrium during left ventricular systole [1-6]. It is well recognized today that a floppy mitral valve (FMV) is the central issue in the MVP-mitral valve regurgitation (MVR) story [2, 7] (fig. 1). The term FMV comes from surgical and pathologic studies and refers to the expansion of the area of the mitral valve leaflets with elongated chordae tendineae, chordae tendineae rupture and often mitral annular dilatation $[2,5,7]$. The prevalence of FMV/MVP as defined above in the general population is $2-3 \%$ [2, 8-13]. It is important to separate studies where the diagnosis was based solely on prolapse of the mitral valve without defining if the mitral valve was 'floppy' or not. It should be noted that MVP may not be a specific finding because it also depends on the left ventricular contractility, left ventricular volume and other ventricular hemodynamics $[1,2]$. FMV consists of a heritable heterogeneous group with at least two forms of inheritance. One form is transmitted by an autosomal dominant inheritance with a variable degree of penetration; this form is the most common type. At present 3 gene loci have been reported (fig. 1). There is another less common form which is transmitted with chromosome X [14-18]. Further, FMV may be isolated or part of a well-recognized

\section{KARGER}

E-Mail karger@karger.com

www.karger.com/crd
Assist. Prof. Konstantinos Dean Boudoulas, MD

Division of Cardiovascular Medicine, The Ohio State University Medical Center 473 West 12th Avenue, Suite 200

Columbus, OH 43220 (USA)

E-Mail kdboudoulas@ osumc.edu 


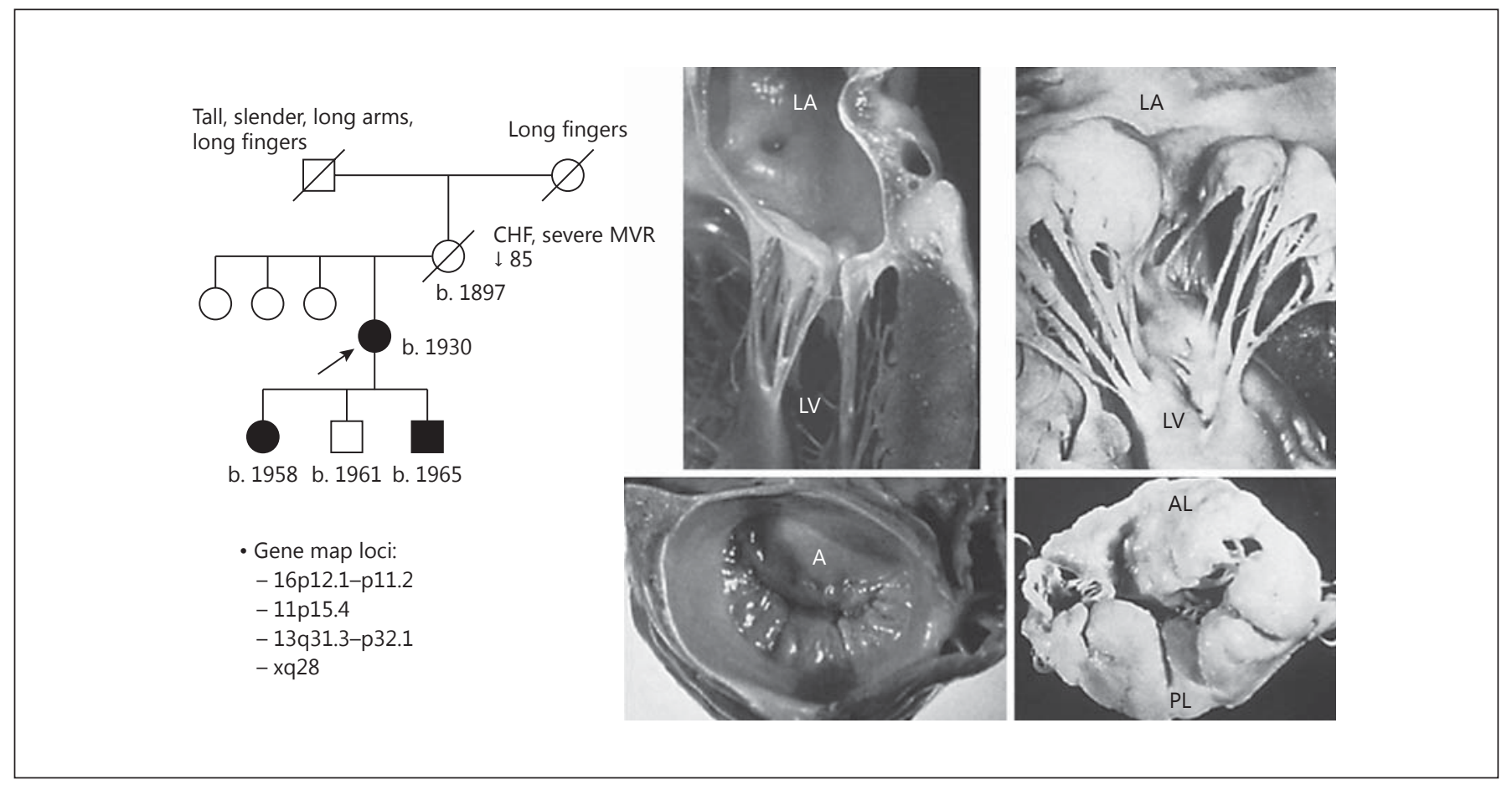

Fig. 1. FMV (far right upper and lower panels) and a normal mitral valve are shown. FMV picture is from Edwards et al. [84]. A fourgeneration pedigree of a family with heritable FMV is also shown (pedigree is from Boudoulas and Wooley [2]). The affected proband is indicated by an arrow. Shaded symbols denote family members with FMV/MVP. Circles represent females and squares represent males. Diagonal slashes through symbols denote deceased members. b. = Year born; $\mathrm{CHF}=$ congestive heart failure; $\mathrm{LA}=$ left atrium; $\mathrm{LV}=$ left ventricle; $\mathrm{AL}=$ anterior leaflet; $\mathrm{PL}=$ posterior leaflet.
Fig. 2. Imaging of FMV/MVP: upper left and upper center panels: three-dimensional echocardiogram; lower left and lower center panels: magnetic resonance imaging. Thick anterior (AL) and posterior leaflets (PL) of the mitral valve are shown; upper right panel: ventriculogram in a patient with FMV/MVP without mitral regurgitation; arrows indicate posterior mitral leaflet prolapse; lower right panel: ventriculogram in a patient with FMV/MVP with severe mitral regurgitation; note the large left atrium (LA) and the thickness of the mitral valve leaflets (arrows; ventriculograms are from Boudoulas and Wooley [2]). Magnetic resonance imaging by courtesy of Subha Raman, MD, The Ohio State University, Columbus, Ohio, USA. LV = Left ventricle; $\mathrm{RV}=$ right ventricle; $\mathrm{Ao}=$ aorta; $\mathrm{LA}=\mathrm{left}$ atrium.
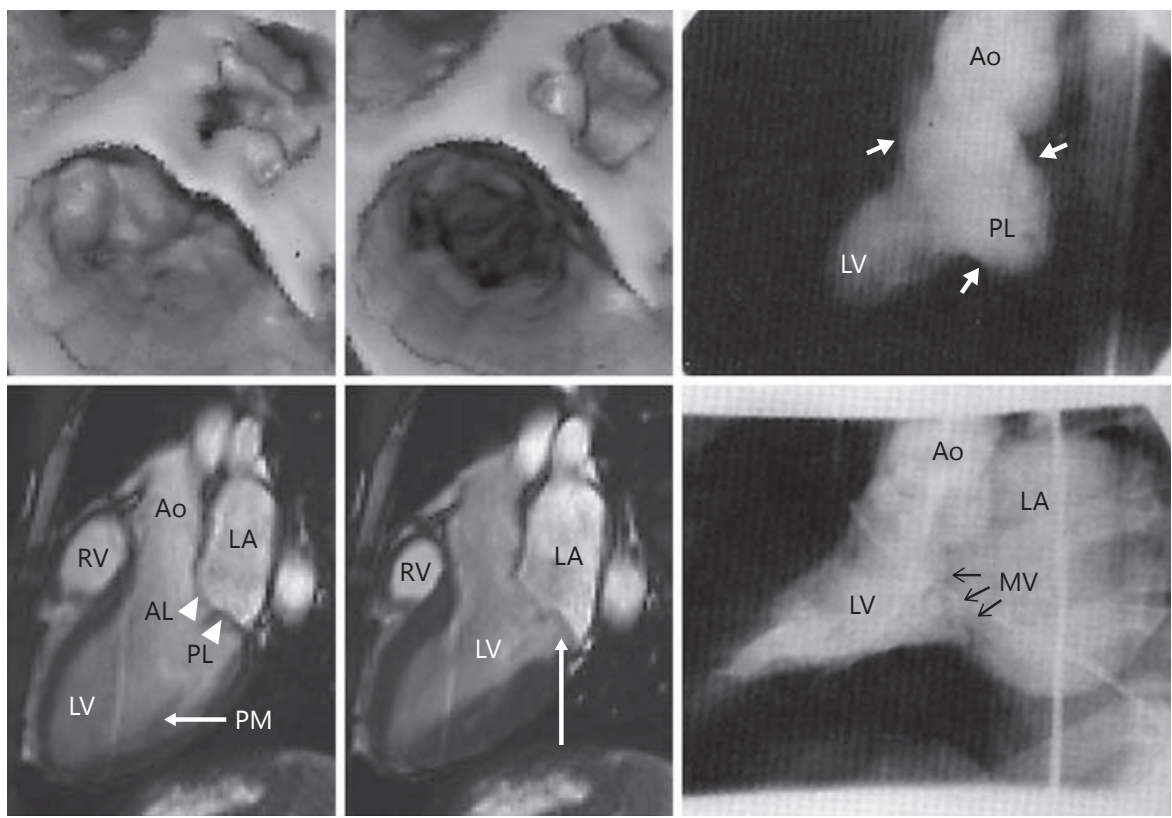


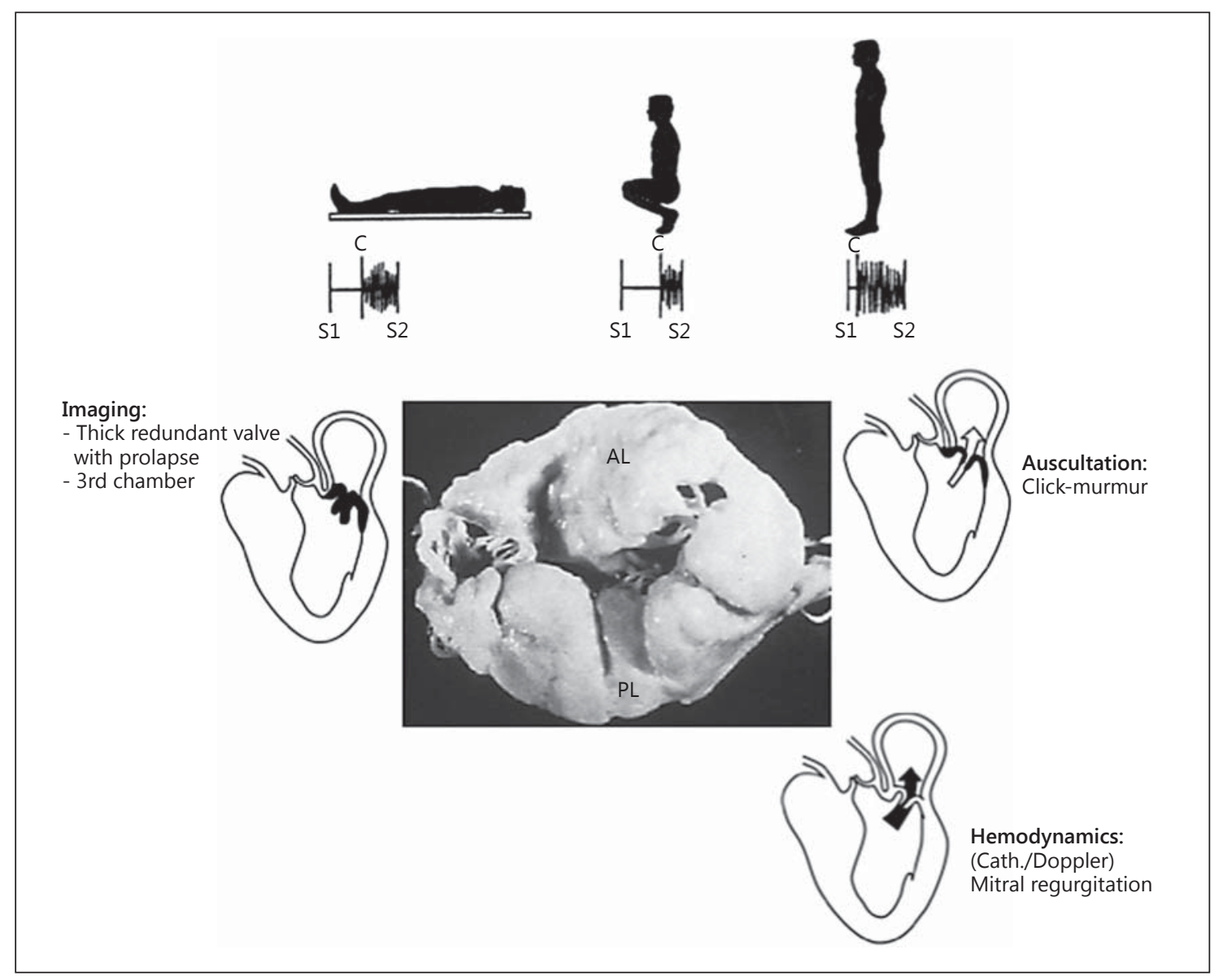

Fig. 3. FMV is the central issue in the FMV/MVP/MVR. Imaging techniques (e.g. echocardiography or magnetic resonance imaging) provide detailed information related to FMV. Hemodynamics (e.g. Doppler or catheterization, Cath.) can demonstrate the severity of MVR; auscultation or a phonocardiogram provides information on the murmur or click. Late systolic murmur with postural changes is also shown. AL = Anterior mitral valve leaflet; $\mathrm{PL}=$ posterior mitral valve leaflet; $\mathrm{C}$ = apical systolic click; $\mathrm{S} 1$ = first heart sound; $\mathrm{S} 2$ = second heart sound [modified from ref. 6].

syndrome of heritable connective tissue disorders such as the Marfan syndrome, Ehlers-Danlos syndrome or polycystic kidney disease [e.g. 19-21]. Isolated FMV/MVP is also considered a cardiovascular abnormality of connective tissue origin. Due to its frequency in the general population, FMV/MVP constitutes the larger group of patients with heritable connective tissue abnormalities of the heart $[1,2,22]$.

\section{Diagnostic Considerations}

The FMV should be the basis for the diagnosis of MVP [1-3] (fig. 2, 3). Auscultatory findings and imaging characteristics are directly related to pathology and function of the mitral valve apparatus $[2,3,5]$. Family history provides important information since the FMV/MVP may be inherited and also may be associated with heritable disorders of connective tissue origin (e.g. Marfan syndrome, Ehlers-Danlos syndrome, or polycystic kidney disease) $[1,2,14,20,21]$. Skeletal abnormalities such as scoliosis, narrow anteroposterior chest diameter, strait back or pectus excavatum, may be found in FMV/MVP. Patients with FMV/MVP are often thinner than normal, have an arm span greater than body height, and their height/weight ratio exceeds that of normal controls [2226].

Cardiac auscultation provides significant information [27-29]. The presence of a non-ejection apical systolic click with or without late apical systolic murmur consti- 
tute the auscultatory criteria for the diagnosis of FMV/ MVP; multiple systolic clicks may be heard. A highpitched mid-to-late apical systolic murmur of mitral regurgitation preceded often by a click, but may occur alone, may also be present. When the posterior leaflet prolapses, the murmur may radiate anteriorly along the left sternal border; when the anterior leaflet prolapses, the murmur may radiate to the axilla and to the spine. The systolic click moves toward the first heart sound with upright posture and the systolic murmur becomes longer and often louder; a systolic murmur may be present only in the upright position. The postural auscultatory changes occurring in patients with FMV/MVP are primarily related to changes in left ventricular volume, myocardial contractility and heart rate (fig. 3). With the progression of disease and when the mitral regurgitation becomes significant, the murmur may become holosystolic and the click may disappear [1, 2, 7, 28, 29].

Echocardiography and Doppler echocardiography is the most widely used and the most useful technique for the diagnostic evaluation and follow-up of patients with FMV/MVP. The echocardiogram will identify the presence and the magnitude of the FMV, the area and the thickness of the mitral leaflets, the mitral annular size, the chordae tendineae length, the severity of mitral regurgitation, pulmonary artery pressure, and its effect on left ventricular and left atrial structure and function. The diagnosis should be based on firm criteria dealing with structural changes and not only on the prolapse, which could be nonspecific [1-3, 30-34]. The introduction of three-dimensional echocardiography in clinical practice provides more precise information related to the structure and function of FMV [34] (fig. 2). Doppler and color Doppler echocardiography provide useful information for the definition and quantification of mitral regurgitation. MVP is usually defined as leaflet(s) displacement at least $2 \mathrm{~mm}$ above the mitral annulus in the parasternal long axis view. Leaflet thickness $\geq 5 \mathrm{~mm}$ is considered abnormal. Echocardiographic criteria of MVP without evidence of mitral valve leaflet thickening require thoughtful analysis [1-3]. In general, however, the diagnosis of FMV/MVP is reliable when it is based on the auscultatory postural complex with confirmatory echocardiographic and Doppler findings. At times, distinguishing between the normal mitral valve and a mitral valve with minor abnormality may be difficult. In certain cases, repeat examination over several years may be necessary to define FMV/MVP since the disease is progressive.
Magnetic resonance imaging can be used in clinical practice for the diagnostic evaluation of patients with FMV/MVP. The method can be applied for follow-up since it is the most accurate method available today to follow changes in left ventricular size, mass and function, to determine left atrial size and function, and the severity of mitral regurgitation. The thickness of the mitral valve can also be defined [35, 36] (fig. 2).

The indications for cardiac catheterization have changed with the development of sophisticated imaging techniques. Ventriculography can define the characteristic appearance of the mitral valve including the thickness of the leaflets (fig. 2). In addition, the severity of mitral regurgitation, left atrial size, and left ventricular size and function can be defined. Intracardiac and pulmonary artery pressure measurements provide important information in certain cases. Coronary arteriography is important to perform prior to surgical intervention in most patients with FMV/MVP $[1,2,5]$.

Surgical inspection of the mitral valve at the time of surgery has contributed to the etiology, pathophysiology and imaging principles already mentioned, and provided the basis for new types of in vivo clinical pathophysiological correlates $[1,2]$.

A dynamic postmortem examination with inspection of the mitral valve from the left atrium before opening the left ventricle and observation of mitral valve dynamics following ventricular filling has provided postmortem observations that paralleled imaging studies and surgical inspection [8]. The characteristic macroscopic appearance of the FMV is easily recognized $[8,37]$; inspection of the mitral valve from the left atrium allows identification of cusp prolapse. After left ventricular opening, the mitral valve leaflet area and chordae tendineae length or rupture can be defined. The pathology of the FMV differs significantly from the normal mitral valve. The most specific histopathologic characteristics are collagen dissolution and disruption in the pars fibrosa of the mitral valve leaflets. Also, there is a replacement of the dense collagenous fibrosa by loose myxomatous connective tissue $[38,39]$. Similar histologic abnormalities have been seen in chordae tendineae. Scanning electron photomicrographs have demonstrated surface folds and focal loss of endothelial cells of mitral valve leaflets, and these surface abnormalities may predispose to thromboembolic complications and infective endocarditis. Continuous pressure and stress due to left ventricular systole on the mitral valve leaflets and chordae tendineae contribute to the gradual progression of these histologic changes $[1,2]$.
72

Cardiology 2013;126:69-80

DOI: $10.1159 / 000351094$
Boudoulas/Boudoulas 
Fig. 4. Upper panel: clinical classification of FMV/MVP. Lower left panel: the dynamic spectrum, time in years and the progression of FMV/MVP are shown. A subtle gradation (gray area) exists between the normal mitral valve and valves with mild FMV/ MVP without MVR. Progression from FMV/MVP with no MVR to another level may or may not occur. Most of the patients with the FMV/MVP syndrome occupy the area above the dotted line, while patients with progressive MVR occupy the area below the dotted line. Lower right panel: the large circle represents the total number of patients with FMV/MVP. Patients with FMV/MVP may be symptomatic or asymptomatic. Symptoms may be directly related to MVR (black circle) or to autonomic dysfunction (gray circle). Certain patients with symptoms related to MVR may also have symptoms secondary to autonomic dysfunction [modified from ref. 1].

\begin{tabular}{|c|c|}
\hline \multicolumn{2}{|c|}{ Classification of FMV/MVP } \\
\hline $\begin{array}{l}\text { FMV/MVP/MVR } \\
\text { - Common mitral valve abnormality with a } \\
\text { spectrum of structural changes, mild to } \\
\text { severe } \\
\text { The basis for: } \\
\text { - Systolic click; mid-late systolic murmur } \\
\text { - Mild or progressive mitral valve dysfunction } \\
\text { - Progressive MVR, atrial fibrillation, } \\
\text { congestive heart failure } \\
\text { - Infective endocarditis } \\
\text { - Embolic phenomena } \\
\text { - Characterized by long natural history } \\
\text { - May be heritable or associated with } \\
\text { heritable disorders of connective tissue } \\
\text { - Conduction system involvement possibly } \\
\text { leading to arrhythmias and conduction } \\
\text { defects }\end{array}$ & $\begin{array}{l}\text { FMV/MVP syndrome } \\
\text { - Patients with FMV/MVP } \\
\text { - Symptoms complex: palpitations, fatigue, exercise } \\
\text { intolerance, dyspnea, chest pain, postural } \\
\text { phenomena, syncope-presyncope, } \\
\text { neuropsychiatric symptoms } \\
\text { - Neuroendocrine or autonomic dysfunction may } \\
\text { provide explanation for symptoms including: } \\
\text { high catecholamine regulation abnormality, } \\
\beta \text {-adrenergic receptor abnormality, hyperresponsive } \\
\text { to adrenergic stimulation, parasympathetic } \\
\text { abnormality, baroreflex modulation abnormality, } \\
\text { renin-aldosterone regulation abnormality, } \\
\text { decreased intravascular volume with upright } \\
\text { posture, atrial natriuretic factor secretion } \\
\text { abnormality } \\
\text { - FMV/MVP - a possible marker for autonomic } \\
\text { dysfunction }\end{array}$ \\
\hline
\end{tabular}

FMV/MVP/MVR: dynamic spectrum and natural progression

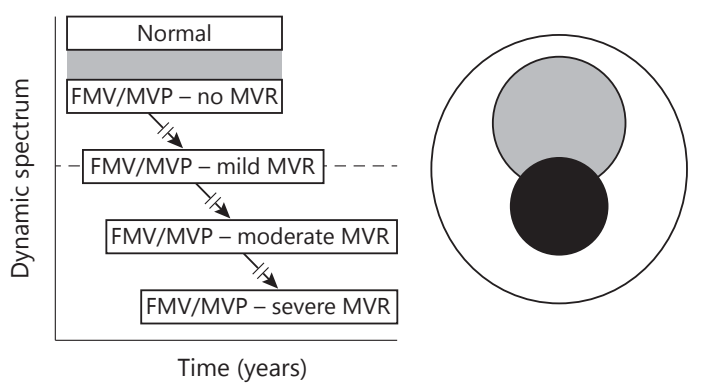

\section{Classification}

FMV/MVP occurs in a heterogeneous group of patients with a wide spectrum of mitral valve involvement and hemodynamic abnormalities from mild to severe. Thus, it is important in each case not only to establish the diagnosis of FMV/MVP, but also to define which abnormalities exist within the spectrum of mitral valve abnormalities and determine their severity $[1,2]$. Mitral valves with diffuse thickening are referred to as Barlow's valve, while patients with regional thickening of the mitral valve are referred to as a fibroelastic deficiency valve. At present, it is not clear if these two entities are genetically different or (most likely) represent a different spectrum of the same disease that encompasses a wide spectrum of abnormalities. For this reason, and to avoid confusion, we believe that it is better to define patients with diffuse or regional mitral valve thickening [1-3].

Two types of symptoms can be defined in FMV/MVP patients (see FMV/MVP/MVR and FMV/MVP syndrome classification for details related to symptoms). In one group of patients, symptoms, physical findings and natural history are directly related to progressive mitral regurgitation and its complications through their effects on left atrial-left ventricular structure and function, and pulmonary circulation. In the other group of patients, symptoms cannot be explained by the degree of MVR alone; activation of the autonomic nervous systems or neuroendocrine dysfunction has been implicated for the explanation of the symptoms in this group of patients. This group is referred to as the FMV/MVP syndrome [1-5] (fig. 4).

\section{FMV/MVP/MVR}

Symptoms and serious complications related to mitral valve dysfunction in patients with FMV/MVP include progressive mitral regurgitation, rupture of chordae tendineae that requires mitral valve surgery, infective endocarditis and thromboembolic phenomena. As a general rule, complications increase with age $[1,2,4,5,31,40-42]$ (fig. 5). 
Fig. 5. FMV, MVP and MVR. Symptoms are plotted against patient age in years. Increased symptoms occur after the age of 50 and are related to progressive MVR, atrial fibrillation, left atrial (LA) and left ventricular (LV) structural and functional changes, and congestive heart failure. Thromboembolic complications, infective endocarditis and cardiac arrhythmias have been reported in a wide range of ages [from ref. 2].

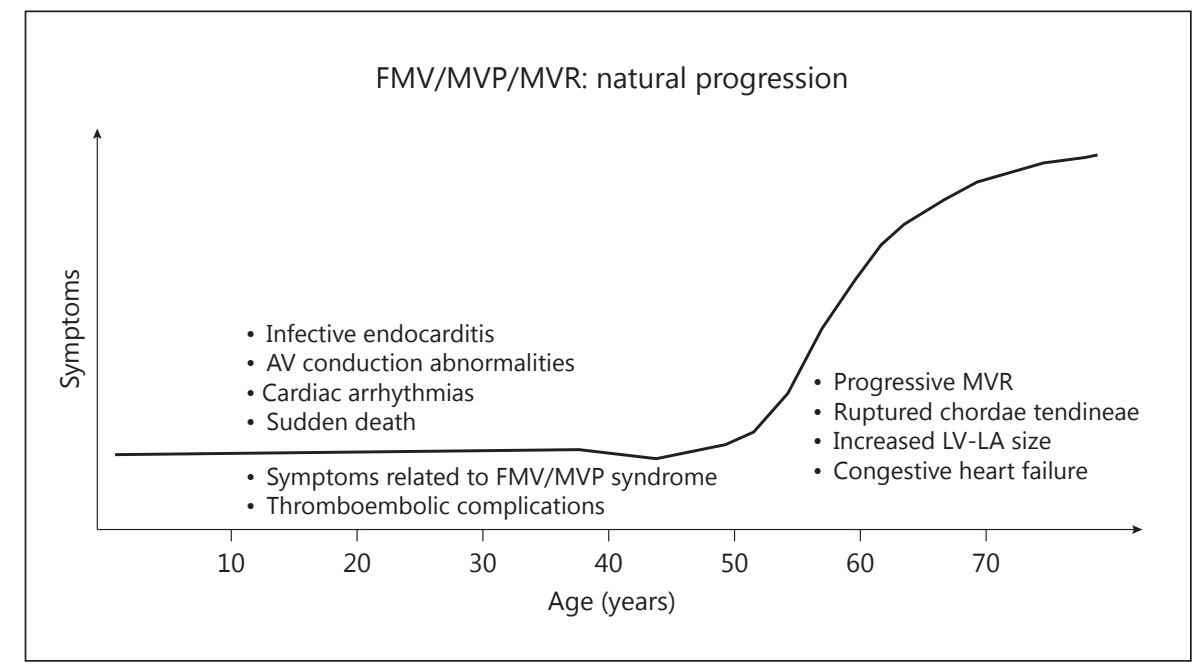

FMV/MVP: Chronic MVR

The progression of MVR from mild to severe in patients with FMV/MVP is generally gradual, and the entire process accelerates after a long asymptomatic period. Significant symptoms typically develop at 50-60 years of age. Progressive mitral regurgitation is related to the various combinations of annular dilatation and elongation or rupture of chordae tendineae; in certain cases, chordae tendineae rupture may lead to acute mitral regurgitation. Patients with thick mitral valve leaflets, mitral systolic murmur, men and those older than 50 years of age are at the highest risk. Progression of mitral regurgitation results in left ventricular dilatation, left atrial enlargement and the development of atrial fibrillation. Left ventricular dilatation may cause papillary muscle displacement, which further exacerbates mitral regurgitation resulting in a vicious cycle. Atrial fibrillation may also increase the severity of mitral regurgitation that improves after restoration of sinus rhythm $[1,2,41-45]$. When FMV/MVP is part of a recognized heritable connective tissue disorder or when it is associated with other cardiovascular abnormalities, the natural history may mostly be related to the underlying disease rather than FMV/MVP per se $[1,2]$.

At present, there are very limited data on the use of pharmacologic agents in an attempt to delay the progression of mitral regurgitation. A study in a small number of patients suggested that therapy with $\beta_{1}$-adrenergic receptor blockade (Toprol XL) over a 2-year period improved left ventricular function in patients with isolated mitral regurgitation [46]. The usefulness of this therapy in clinical practice remains to be defined. Further, attenuation of transforming growth factor- $\beta$ (has a fibrotic effect in the pathogenesis of sporadic MVP) signaling by angiotensin II receptor blockers may prove to be effective in modulating the pathological progression of MVP in these patients $[4-6,47]$. Elastic properties of the aorta decrease with age (i.e. the aorta becomes stiffer). Stiffening of the aorta with age may increase the degree of mitral regurgitation and precipitate the natural history of the disease. Determination of aortic function (e.g. pulse wave velocity or dispensability) may help to better define the natural history of FMV/MVP. Since a stiff aorta is associated with systolic arterial hypertension, better control of systolic hypertension, especially with drugs that improve aortic function such as angiotensin-converting enzyme inhibitors or calcium channel blockers, may prove to slow the progression of the disease; however, this remains to be defined $[1,2]$.

Infective endocarditis is a complication of FMV/MVP. Antibiotic prophylaxis for infective endocarditis is currently a matter of considerable debate, as well a subject of changing attitudes and recommendations. Patients should be encouraged to maintain the best possible oral hygiene to reduce potential sources of bacterial seeding. While at present guidelines do not recommend prophylaxis for endocarditis in patients with FMV/MVP, it should be noted that the abnormal area of valve leaflets and not the mitral regurgitation per se is responsible of endocarditis $[5,48,49]$.

Thromboembolic complications are another controversial issue. Patients who have had clinical manifestation of retinal, cerebral or peripheral emboli should discontinue oral contraceptives, abstain from cigarette smoking and have a careful cardiovascular and hematological eval- 
uation before long-term anticoagulation or antiplatelet therapy is considered. Chronic anticoagulation should be considered in patients with chronic atrial fibrillation with or without thromboembolic phenomena as is indicated in patients without FMV/MVP [1, 2, 50, 51].

Cardiac arrhythmias appear to be more frequent in patients with FMV/MVP compared to the general population. The same diagnostic and therapeutic approach should be used as in patients without FMV/MVP. Sudden cardiac death in patients with FMV/MVP in the absence of significant mitral regurgitation may occur but is rare. Autopsy studies in patients with FMV/MVP have shown that mitral valve leaflet length and posterior leaflet thickness were greater in hearts from patients who died suddenly compared to patients with FMV/MVP that died from other causes. Patients with FMV/MVP who died suddenly without significant mitral regurgitation tend to be relatively young women. Ventricular fibrillation appears to be the cause of sudden death. $\beta$-Blockade therapy may be beneficial in patients with FMV/MVP who were successfully resuscitated from cardiac arrest $[1,2,52-56]$.

When significant mitral regurgitation is present in a symptomatic patient with FMV/MVP or with a new onset of atrial fibrillation, surgical intervention is recommended. Left ventricular ejection fraction $<60 \%$ or left ventricular end-systolic diameter $>45 \mathrm{~mm}$ are also indications for surgery. An exercise tolerance test may be useful to define if patients are truly asymptomatic or if they develop pulmonary hypertension (systolic pressure $>60$ $\mathrm{mm} \mathrm{Hg}$ ). Studies, however, have shown superior recovery of long-term left ventricular ejection fraction following early mitral valve repair when left ventricular ejection fraction is $\geq 65 \%$ or left ventricular systolic diameter is $<40 \mathrm{~mm}$. Left ventricular ejection fraction is overestimated in mitral regurgitation because of a significant decrease in afterload and an increase in preload. Left atrial volume and function may also help to define the best time for surgical intervention. Reconstructive surgery is preferable and is feasible in the greater proportion of patients with FMV/MVP. The long-term results from reconstructive surgery are excellent [57-69]. Mitral valve annular disjunction is a common component of the mitral valve apparatus in advanced stages of FMV/MVP. Its recognition on transesophageal echocardiography is important to facilitate optimal mitral valve repair $[59,64]$.

\section{FMV/MVP: Acute MVR}

The most common causes of acute mitral regurgitation are chordae tendineae rupture, papillary muscle rupture and infective endocarditis. Distinguishing the three most common types of acute mitral regurgitation based on clinical evaluation is relatively easy $[2,5]$. Rupture of chordae tendineae is the most common cause of acute mitral regurgitation in patients with FMV/MVP. In severe acute mitral regurgitation, a large volume of blood is injected into the low pressure left atrium during left ventricular systole. Since there is insufficient time to allow dilatation of the left ventricle and the left atrium, the large regurgitant volume will result in marked elevation in left atrial pressure. Left atrial $\mathrm{V}$-waves $\geq 60 \mathrm{~mm}$ $\mathrm{Hg}$ is not uncommon. The marked increase in pulmonary venous pressure will result in pulmonary edema. Since the left ventricle is of normal size and a large amount of blood goes into the left atrium during left ventricular systole, the forward stroke volume is markedly decreased. The net result, in addition to pulmonary congestion, is tissue hypoperfusion and shock. The systolic murmur usually peaks in mid-systole and diminishes in intensity before the second heart sound. In cases of posterior chordae tendineae rupture, the murmur may radiate anteriorly to the sternum, while in cases of anterior chordae rupture the murmur may radiate posteriorly to the spine. The sudden appearance of a new systolic murmur associated with dyspnea and tissue hypoperfusion in a middle-aged patient should raise suspicion of acute severe mitral regurgitation. In cases with a known murmur, the patient presents with acute dyspnea. In cases of acute severe mitral regurgitation, emergent surgical intervention is indicated. Patients with less severe mitral regurgitation may progress gradually and may respond to medical management without surgery at least in the acute phase $[2,5]$.

\section{FMV/MVP Syndrome}

Certain patients with FMV/MVP may have symptoms that cannot be explained on the basis of mitral regurgitation alone. Neuroendocrine and/or autonomic nervous system dysfunction is implicated for the explanation of the symptoms in this group of patients. Whether the FMV/MVP syndrome exists has been often debated in the past. Based on our experience and the experience of others, it is our opinion that certain patients with FMV/MVP have symptoms which cannot be explained by the degree of mitral regurgitation. Symptoms in these patients are common and it is thus difficult to see them as a coincidence. Also, several pathophysiologic mechanisms provide an explanation for these symptoms [70-75]. The true incidence of symptoms is not known; the incidence may be exaggerated because most of the studies have been conducted in academic medical centers and may reflect 


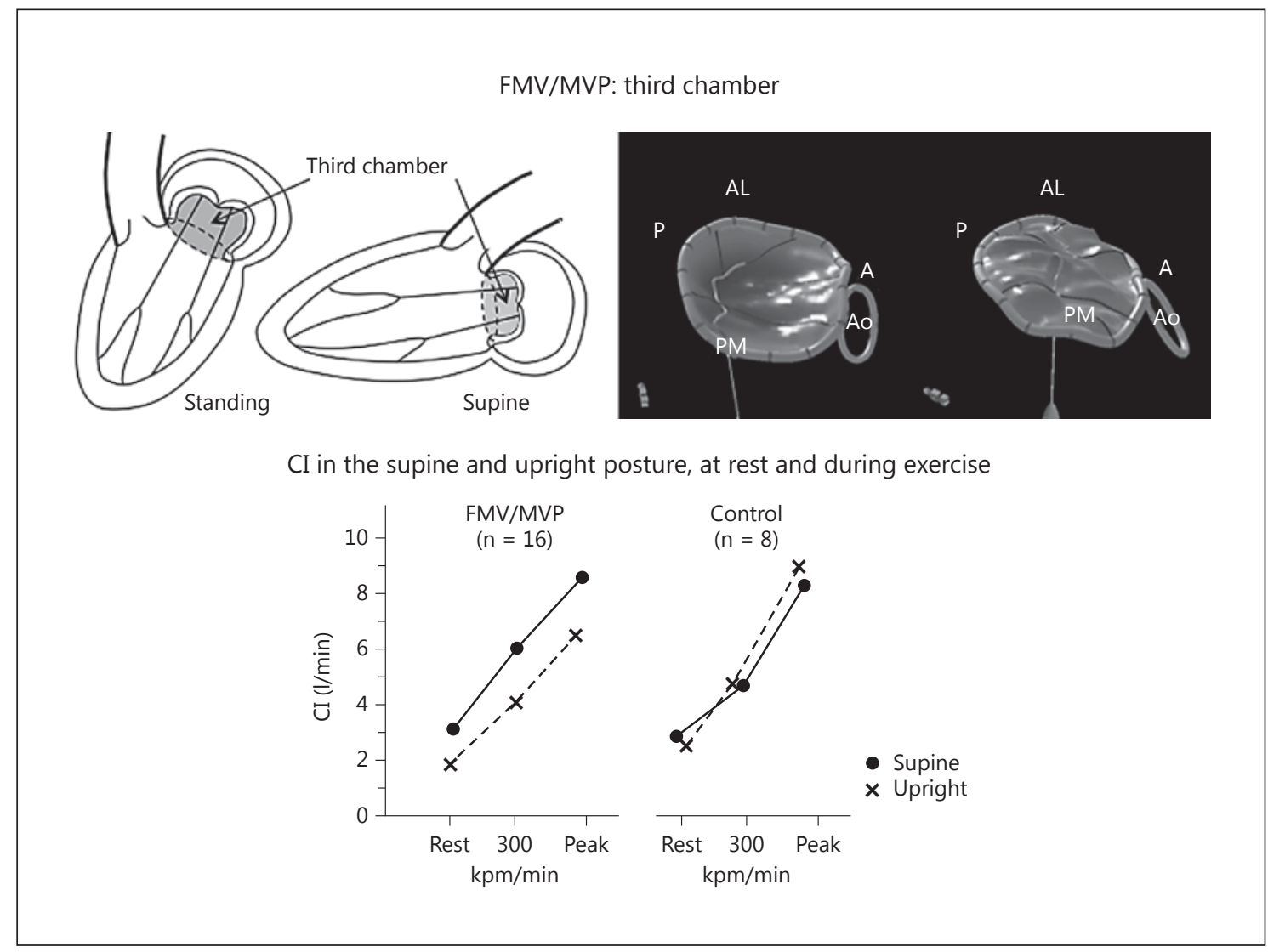

Fig. 6. Upper left panel: the third chamber, the space between the mitral valve annulus and the prolapsing mitral valve leaflets, is shown schematically. Note that the size of the third chamber increases in the upright position. Upper right panel: three-dimensional echocardiogram (courtesy of Vlasis Ninios, St Lucas Hospital Thessaloniki, Greece) showing FMV/MVP and the third chamber (right part of the figure) compared to a normal mitral valve (left part of the figure). $\mathrm{A}=$ Anterior; $\mathrm{AL}=$ anterior leaflet; $\mathrm{Ao}=$ aorta; $\mathrm{P}=$ posterior; $\mathrm{PM}=$ posterior papillary muscle. Lower panel: cardiac index (CI) in patients with the FMV/MVP syndrome was less in the upright compared to the supine position, while in normal subjects CI was similar in the supine and the upright position [modified from ref. 2, 76]. selection bias. The most common symptoms include palpitations, orthostatic phenomena (orthostatic tachycardia, hypotension and arrhythmias), syncope or presyncope, fatigue-exercise intolerance, chest pain and dyspnea. The onset of symptoms encompasses a wide age range with a median of approximately 30 years of age. The symptoms may last for years and occasionally may persist after valve surgery $[2,5]$.

\section{Pathogenetic Mechanisms}

Prolapse of the FMV is a situation in which a redundant mitral valve leaflet(s) prolapses into the left atrium during left ventricular systole. As a result, a number of dynamic events are set in motion. The prolapsing FMV becomes a space-occupying lesion into the left atrium, which results in the development of a third chamber within the border of the mitral valve annulus and the prolapsing mitral valve leaflets [2] (fig. 6). Thus, during left ventricular systole, the left heart consists of three chambers: left ventricle, left atrium and third chamber. Physiologically, the third chamber acts like a left ventricular aneurysm since blood within the space of this chamber does not contribute to the effective stroke volume. There is not enough information today related to the volume of the third chamber. In extreme cases, there may be $\geq 10 \mathrm{ml}$; however, in the majority of cases, it appears to be 3-5 $\mathrm{ml}$. The degree of prolapse increases in the upright position, which may result in a greater decrease in the effective stroke volume and forward cardiac output. Postural exercise studies from our laboratory have shown that control 
Fig. 7. The prolapsing mitral valve becomes a space-occupying lesion of the left atrial (LA) chamber and may stimulate neurohumoral activation. FMV is shown in the middle [from ref. 84]; FMV innervation is shown schematically above; upper right shows papillary muscle traction during left ventricular (LV) systole; lower right shows stretch-activated receptor schematically; left upper shows interaction between the brain, heart, kidneys and adrenals; lower left shows $\beta$-adrenergic receptor schematically; lower panel shows orthostatic phenomena [modified from ref. 52].

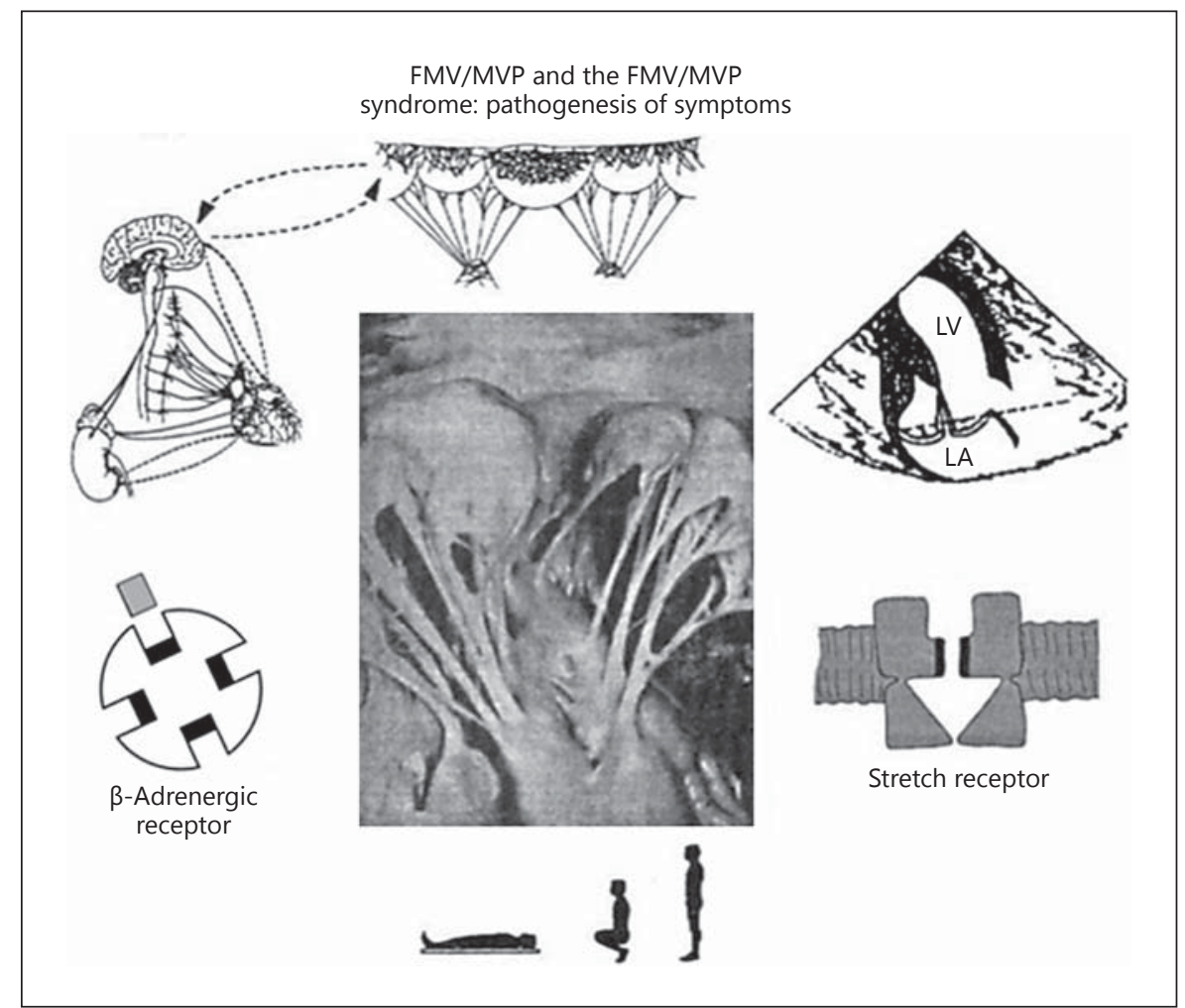

subjects exercised longer and achieved a greater workload in both the supine and upright positions than FMV/MVP syndrome patients. FMV/MVP syndrome patients consistently exhibited a smaller left ventricular end-diastolic volume and cardiac output in the upright position compared to supine, and this difference was maintained throughout the exercise period. In contrast, control subjects exhibited a fall in the left ventricular end-diastolic volume, but by the time peak exercise was achieved, the upright volumes were quite similar to the supine volumes [76]. These findings may partially explain the orthostatic phenomena, fatigue and exercise intolerance. A decrease in ventricular volume may also stimulate the adrenergic nervous system.

The prolapsing mitral valve results in traction of the papillary muscles (fig. 7). Papillary muscle traction may result in left ventricular contraction and relaxation abnormalities and activation of stretch receptors. Stretch receptor activation may result in membrane depolarization and cardiac arrhythmias [77-79].

The human mitral valve has distinct patterns of innervation. Mechanical stimuli caused by abnormal coaptation of FMV/MVP may cause an abnormal autonomic nerve feedback between the central nervous system and the mitral valve $[2,52]$ (fig. 7). Patients with the FMV/ MVP syndrome with normal left ventricular size and function, and normal left atrial size without significant mitral regurgitation and heart failure had higher 24-hour urinary epinephrine and norepinephrine excretion compared to normal controls (fig. 8). In addition, the frequency of premature ventricular beats detected in these patients by ambulatory monitoring paralleled urine catecholamine excretion. Catecholamines and premature ventricular beats decreased significantly during the night; the supine position may also have contributed to a decrease in catecholamines. When 24-hour urine epinephrine and norepinephrine was measured over 3 consecutive days, there was no day-to-day variability in the catecholamine levels [80].

An increase in the adrenergic tone can be associated with shortening of left ventricular systole. Increased adrenergic tone in patients with the FMV/MVP syndrome prompted a study to determine the response to adrenergic stimulation. During isoproterenol infusion, there were no control subjects who developed symptoms, excluding palpitations. In contrast, isoproterenol infusion at $0.5-2.0 \mu \mathrm{g} / \mathrm{min}$ in patients with the FMV/MVP syndrome reproduced symptoms in a dose-related manner. 

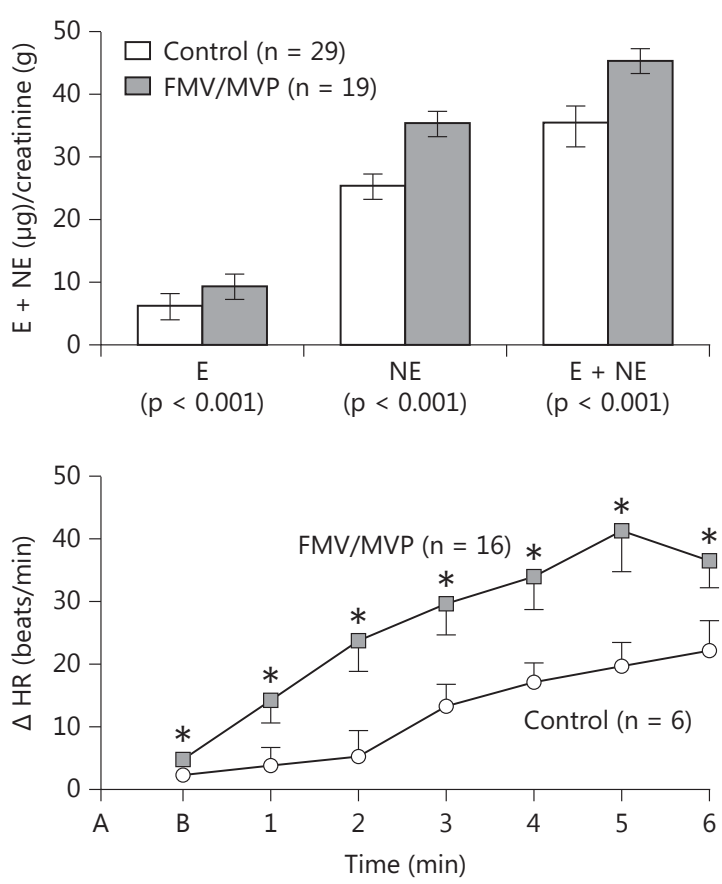

Fig. 8. Upper panel: 24-hour urinary epinephrine (E) and norepinephrine (NE) excretion. Patients with the FMV/MVP syndrome had higher levels compared to normal subjects (control [from ref. $80])$. Lower panel: changes in heart rate (HR) during isoproterenol infusion $(2 \mu \mathrm{g} / \mathrm{min})$ in patients with the FMV/MVP syndrome and controls [modified from ref. 81]. *Statistically significant.

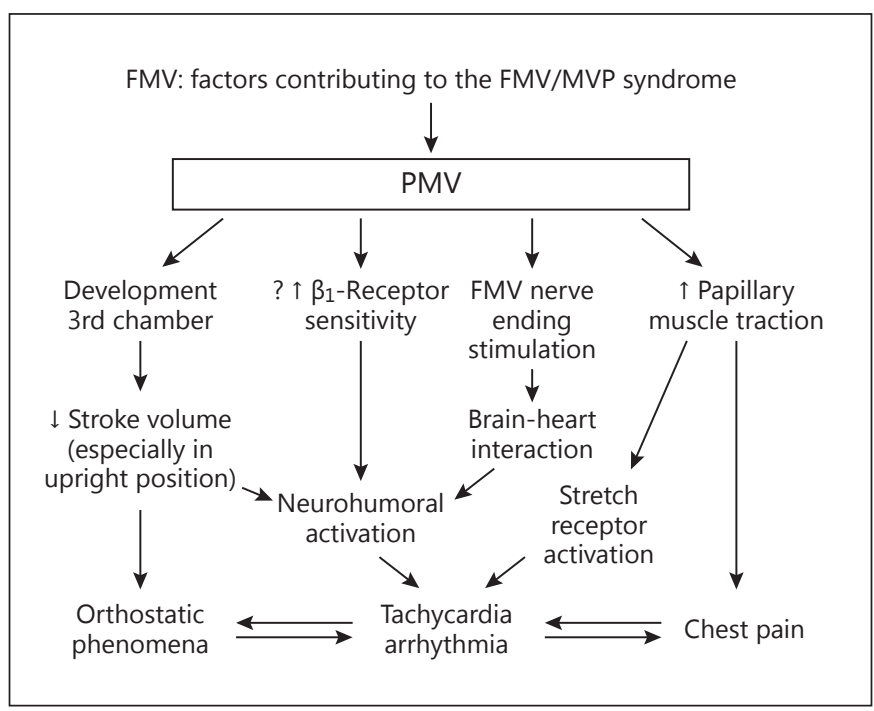

Fig. 9. FMV: factors contributing to the pathogenesis of the FMV/ MVP syndrome; schematic presentation [modified from ref. 2].
Symptoms that developed during isoproterenol infusion included chest pain, extreme fatigue, dyspnea, dizziness and panic attacks after infusion. In addition, the increase in heart rate during isoproterenol infusion was significantly greater in patients with the FMV/MVP syndrome compared to controls [81] (fig. 8). Hyperresponses to isoproterenol may be related to an increased sensitivity of $\beta_{1}$-adrenergic receptors. High adrenergic activity is usually associated with lower serum potassium, which may contribute to fatigue and arrhythmias [82, 83]. Figure 9 shows the pathophysiologic mechanisms related to the FMV/MVP syndrome schematically.

\section{General Principles of Management}

Careful explanation of clinical findings to patients along with current knowledge related to possible mechanisms of symptoms will provide a foundation for longterm management. Importantly, patients with the FMV/ MVP syndrome should be protected from unnecessary surgery if they do not have severe mitral regurgitation. There are no studies related to the prognosis in patients with the FMV/MVP syndrome; however, symptoms may persist for years and in certain cases may continue to be present after valve surgery. Prognosis as far as life expectancy appears to be quite good. Patients with the FMV/ MVP syndrome seem to be sensitive to volume depletion and postural changes. They should be advised to avoid or discontinue chronic diuretic therapy. Fluid intake before, during and after exercise could be beneficial especially in patients with low intravascular volume. An important measure is to avoid catecholamine and cyclic AMP stimulants by abstaining from caffeine, cigarettes, alcohol and over-the-counter drugs containing epinephrine or ephedrine. Low doses of $\beta$-blockers over a short period of time during stressful periods may be beneficial. Studies suggest that aerobic exercise may give good results in certain patients. At present, there is insufficient information for the use of Procoralan in case of orthostatic tachycardia in patients with the FMV/MVP syndrome $[2,75]$.

\section{Individual Patient Analysis}

Individual patient analysis in a heterogeneous group of patients with FMV/MVP or the FMV/MVP syndrome presents a logical approach to the diagnostic and therapeutic process. Emphasis should be placed on the individual patient profile. Diagnostic and therapeutic methods vary and depend on the physician's experience, facilities available and technology. Facilities vary from one 
institution to another and definitely will change in the years to come. Specific definition of the anatomic lesions, physiologic state, pathophysiologic abnormalities and eventually genetic testing in the future should be a standard approach [2].

\section{Acknowledgment}

We are pleased to acknowledge the late Charles F. Wooley, MD, superior physician, outstanding educator, mentor, colleague and friend, for his significant contributions in the area of FMV/MVP.

\section{References}

1 Boudoulas H, Wooley CF (eds): Mitral Valve Prolapse and the Mitral Valve Prolapse Syndrome. Mount Kisco, Futura, 1998.

2 Boudoulas H, Wooley CF (eds): Mitral Valve: Floppy Mitral Valve, Mitral Valve Prolapse, Mitral Valvular Regurgitation, ed 2, revised. Armonk, Futura, 2000.

-3 Boudoulas KD, Boudoulas H: Floppy mitral valve and mitral valve prolapse: lack of precise definition (the tower of Babel syndrome) Cardiology 2011;118:93-96.

4 Boudoulas H, Kolibash AJ, Baker P, et al: Mitral valve prolapse and the mitral valve prolapse syndrome: a diagnostic classification and pathogenesis of symptoms. Am Heart J 1989;118:796-818.

5 Boudoulas H: Valvular Disease: American College of Cardiology Self-Assessment Program (ACCSAP) 2009.

6 Wooley CF, Sparks EA, Boudoulas H: The floppy mitral valve-mitral valve prolapse-mitral valvular regurgitation triad. ACC Current J Rev 1994, pp 25-26.

7 Wooley CF, Baker PB, Kolibash AJ, et al: The floppy, myxomatous mitral valve and mitral regurgitation. Prog Cardiovasc Dis 1991;33: 397-433.

$\checkmark 8$ Davies MJ, Moore BP, Brainbridge MV: The floppy mitral valve: study of incidence, pathology, and complications in surgical, necropsy and forensic material. Br Heart J 1978; 40:468-481.

$\checkmark 9$ Freed LA, Levy D, Levine RA, et al: Prevalence and clinical outcome of mitral valve prolapse. N Engl J Med 1999;341:1-7.

10 Hickey AJ, Wolfers J, Wilcken DE: Mitral valve prolapse: prevalence in an Australian population. Med J Aust 1981;1:31-33.

11 Savage DD, Garisson RJ, Devereux RB, et al: Mitral valve prolapse in the general population. 1. Epidemiologic features: the Framingham Study. Am Heart J 1983;106:571-576.

$\checkmark 12$ Ohara N, Mikajima T, Kato H: Mitral valve prolapse in childhood: the incidence and clinical presentation in different age groups. Acta Paediatr Jpn 1991;33:467-475.

13 Warth DC, King ME, Cohen JM, et al: Prevalence of mitral valve prolapse in normal children. J Am Coll Cardiol 1985;5:117-125.

14 Devereux RB, Brown WT, Kramer-Fox R, et al: Inheritance of mitral valve prolapse: effect of age and sex on gene expression. Ann Intern Med 1982;97:826-832.
15 KontopoulosAG, Harsoulis P, Adam K, et al: Frequency of HLA antigens in Graves' hyperthyroidism and mitral valve prolapse. J Heart Valve Dis 1996;5:543-545.

16 Grau JB, Pirelli L, Yu P-J, et al: The genetics of mitral valve prolapse. Clin Genet 2007;72: 288-295.

17 Kyndt F, Gueffet JP, Probst V, et al: Mutations in the gene encoding filamin $\mathrm{A}$ as a cause for familial cardiac valvular dystrophy. Circulation 2007;115:40-49.

18 Rizzon P, Biasco G, Brindicci G, et al: Familial syndrome of midsystolic click and late systolic murmur. Br Heart J 1973;35:245-259.

19 Bowen J, Boudoulas H, Wooley CF: Cardiovascular disease of connective tissue origin. Am J Med 1987;82:481-488.

20 Leier CV, Call TD, Fulkerson PK, et al: The spectrum of cardiac defects in the EhlersDanlos syndrome, types I and III. Ann Intern Med 1980;92:171-178.

21 Hirata K, Triposkiadis F, Sparks E, et al: The Marfan syndrome: cardiovascular physical findings and diagnostic correlates. Am Heart J 1992;123:743-752.

22 Glesby MJ, Pyeritz RE: Association of mitral valve prolapse and systemic abnormalities of connective tissue: a phenotypic continuum. JAMA 1989;262:523-528.

23 Devereux RB, Brown WT, Lutas EM, et al: Association of mitral valve prolapse with low body weight and low blood pressure. Lancet 1982;ii:792-795.

24 Udoshi MB, Shah A, Fisher VJ, et al: Incidence of mitral valve prolapse in subjects with thoracic skeletal abnormalities - a prospective study. Am Heart J 1979;97:303-311.

25 Schutte JE, Gaffney FA, Blend L, et al: Distinc tive anthropometric characteristics of women with mitral valve prolapse. Am J Med 1981;71: 533-538.

26 Rosenberg CA, Derman GH, Grabb WC, et al: Hypomastia and mitral valve prolapse. $\mathrm{N}$ Engl J Med 1983;309:1230-1232.

-27 Fontana NE, Sparks EA, Boudoulas H, et al: Mitral valve prolapse and the mitral valve prolapse syndrome. Curr Probl Cardiol 1991; 16:311-375.

28 Barlow JB, Pocock WA, Marchand P, et al: The significance of late systolic murmurs. Am Heart J 1963;66:443-452.

29 Criley JM, Kissel GL: Prolapse of the mitral valve: the click and late systolic murmur syndrome. Progr Cardiovasc Dis 1975;4:23-36.
30 Malkowski MT, Boudoulas H, Wooley CF, et al: The spectrum of structural abnormalities in the floppy mitral valve: echocardiographic evaluation. Am Heart J 1996;132:145-151.

31 Nishimura RA, McGoon MD, Shub C, et al: Echocardiographically documented mitral valve prolapse. Long-term follow-up of 237 patients. N Engl J Med 1985;313:1303-1309.

32 Avierinos JF, Inamo J, Gligioni F, et al: Sex differences in morphology and outcomes of mitral valve prolapse. Ann Intern Med 2008; 149:787-794.

33 Thavendiranathan P, Phelan D, Thomas JD: Quantitative assessment of mitral regurgitation; validation of new methods. J Am Coll Cardiol 2012;60:1470-1483.

-34 Lang RM, Tsang W, Weinert L, et al: Valvular heart disease. The value of 3-dimensional echocardiography. J Am Coll Cardiol 2011; 58:1933-1944.

-35 Maron MS, Olivotto I, Harrigan C, et al: Mitral valve abnormalities identified by cardiovascular magnetic resonance represent a primary phenotypic expression of hypertrophic cardiomyopathy. Circulation 2011;124:40-47.

36 Azevedo CF, Nigri M, Higuchi ML, et al: Prognostic significance of myocardial fibrosis quantification by histopathology and magnetic resonance imaging in patients with severe aortic valve disease. J Am Coll Cardiol 2012;56:278-287.

37 Pomerance A: Ballooning deformity (mucoid degeneration) of atrioventricular valves. $\mathrm{Br}$ Heart J 1969;31:343-351.

38 King BD, Clark MA, Baba N, et al: Myxomatous mitral valves: collagen dissolution as the primary defect. Circulation 1982;66:288-296.

39 Baker PB, Bansal G, Boudoulas H, et al: Floppy mitral valve chordae tendineae: histopathologic alterations. Human Pathol 1988;19: 507-512.

40 Wilcken DEL, Hickey AJ: Lifetime risk for patients with mitral valve prolapse of developing severe valve regurgitation requiring surgery. Circulation 1988;78:10-14.

41 Kolibash AJ, Kilman JW, Bush CA, et al: Evidence for progression from mild to severe mitral regurgitation in mitral valve prolapse. Am J Cardiol 1986;58:762-767.

42 You-Bing D, Takenata K, Sakamoto T, et al: Follow-up in mitral valve prolapse by phonocardiography, M-mode and two-dimensional echocardiography and Doppler echocardiography. Am J Cardiol 1990;65:349-354. 
43 Shah PM, Pravin M: Update of mitral valve prolapse syndrome: when is echo prolapse a pathological prolapse? Echocardiography 1984;1:87-95.

-44 Otani K, Takeuchi M, Kaku K, et al: Evidence of a vicious cycle in mitral regurgitation with prolapse. Circulation 2012;126(suppl 1): S214-S221.

45 Gertz ZM, Raina A, Saghy L, et al: Evidence of atrial functional mitral regurgitation due to atrial fibrillation. J Am Coll Cardiol 2011;58: 1474-1481.

-46 Ahmed MI, Aban I, Lloyd SG, et al: A randomized controlled phase IIb trial of beta(1)receptor blockade for chronic degenerative mitral regurgitation. J Am Coll Cardiol 2012; 60:833-838.

47 Geirsson A, Singh M, Ali R, et al: Modulation of transforming growth factor- $\beta$ signaling and extracellular matrix production in myxomatous mitral valves by angiotensin II receptor blockers. Circulation 2012;126(suppl 1):S189-S197.

48 Lachmam AS, Bramwell-Jones DM, Lakier $J B$, et al: Infective endocarditis in the billowing mitral leaflet syndrome. Br Heart J 1975; $37: 226-230$

-49 Danchin N, Briancon S, Mathieu P, et al: Mitral valve prolapse as a risk factor for infective endocarditis. Lancet 1989;i:743-745.

-50 Barnett HJM, Jones MW, Boughner DR, et al: Cerebral ischemic events associated with prolapsing mitral valve. Arch Neurol 1976;33: 777-782.

-51 Elam MB, Viar MJ, Ratts TE, et al: Mitral valve prolapse in women with oral contraceptiverelated cerebrovascular insufficiency. Associated persistent hypercoagulable state. Arch Intern Med 1986;146:73-77.

52 Boudoulas H, Schaal SF, Wooley CF: Floppy mitral valve/mitral valve prolapse: cardiac arrhythmias; in Vardas PE (ed): Cardiac Arrhythmias, Pacing, and Electrophysiology. Dordrecht, Kluwer Academic, 1998, pp 8995.

53 Boudoulas H, Schaal SF, Stang JM, et al: Mitral valve prolapse: cardiac arrest with longterm survival. Int J Cardiol 1990;26:37-44.

54 Winkle RA, Lopes MG, Popp RL, et al: Life threatening arrhythmias in the mitral valve prolapse syndrome. Am J Med 1976;60:961967.

55 Farb A, Tang AL, Atkinson JB, et al: Comparison of cardiac findings in patients with mitral valve prolapse who die suddenly to those who have congestive heart failure from mitral regurgitation and to those with fatal noncardiac conditions. Am J Cardiol 1992;70: 234-239.
6 Chesler E, King RA, Edwards JE: The myxomatous mitral valve and sudden death. Circulation 1983;67:632-639.

57 Cohn LH, Couper GS, Aranki SF, et al: Longterm results of mitral valve reconstruction for regurgitation of the myxomatous mitral valve. J Thorac Cardiovasc Surg 1994;107: 143-159.

58 Enriquez-Sarano M, Schaff HV, Frye RL: Early surgery for mitral regurgitation. The advantages of youth. Circulation 1997;96:41214123.

59 Hutchins GM, Moore GW, Skoog DK: The association of floppy mitral valve with disjunction of the mitral annulus fibrosus. $\mathrm{N}$ Engl J Med 1986;314:535-540.

60 Adams DH, Rosenhek R, Falk V: Degenerative mitral regurgitation: best practice revolution. Eur Heart J 2010;3:1958-1966.

61 Glower DD: Surgical approaches to mitral regurgitation. J Am Coll Cardiol 2012;60:13151322.

62 Le Tourneau T, Messika-Zeitoun D, Russo A, et al: Impact of left atrial volume on clinical outcome in organic mitral regurgitation. J Am Coll Cardiol 2010;56:570-578.

63 Boudoulas KD, Sparks EA, Wooley CF, et al: Factors determining left atrial kinetic energy in patients with chronic mitral valve disease. Herz 2003;28:437-444.

64 Ericson MJ, Bitkover CY, Omran AS, et al: Mitral annular disjunction in advanced myxomatous mitral valve disease: echocardiographic detection and surgical correction. J Am Soc Echocardiogr 2005;18:1014-1022.

65 Bonow RO: Chronic mitral regurgitation and aortic regurgitation: have indications for surgery changed? J Am Coll Cardiol 2013;61: 693-701.

66 Maisano F, La Canna G, Colombo A, et al: The evolution from surgery to percutaneous mitral valve interventions. J Am Coll Cardiol 2011;58:2174-2182.

67 Feldman T, Foster E, Glower DG, et al: Percutaneous repair or surgery for mitral regurgitation. N Engl J Med 2011;364:1395-1406.

68 Whitlow PL, Feldman T, Pedersen W, et al: Acute and 12-month results with catheterbased mitral valve leaflet repair. J Am Coll Cardiol 2012;59:130-139.

69 Magne J, Lancellotti P, Pierard LA: Exerciseinduced changes in degenerative mitral regurgitation. J Am Coll Cardiol 2010;56:300309.
70 Gafney EA, Bastian BC, Lane LB, et al: Abnormal cardiovascular regulation in the mitral valve prolapse syndrome. Am J Cardiol 1983; 52:316-329.

71 Gaffney AF, Karlsson ES, Campbell W, et al: Autonomic dysfunction in women with mitral valve prolapse syndrome. Circulation 1979;59:894-901.

72 Santos AD, Mathew PK, Hilal H: Orthostatic hypotension: a commonly unrecognized cause of symptoms in mitral valve prolapse. Am J Med 1981;71:746-750.

73 Coghlan HC, Phares P, Crowley M, et al: Dysautonomia in mitral valve prolapse. Am J Med 1979;67:236-244.

74 Boudoulas H, Wooley CF: Mitral valve prolapse syndrome: evidence of autonomic dysfunction. J Cardiol 1987;17:3-12.

75 Boudoulas H: Mitral valve prolapse: etiology, clinical presentation and neuroendocrine function. J Heart Valve Dis 1992;1:175-188.

76 Bashore TM, Grines C, Utlak D, et al: Postural exercise abnormalities in symptomatic patients with mitral valve prolapse. J Am Coll Cardiol 1988;11:499-507.

-77 Sanfilippo AJ, Harrigan P, Popvic AD, et al: Papillary muscle tension in mitral valve prolapse. Quantitation by two-dimensional echocardiography. J Am Coll Cardiol 1992; 19:564-571.

78 Franz MR, Cima R, Wang D, et al: Electrophysiological effects of myocardial stretch and mechanical determinants of stretch-activated arrhythmias. Circulation 1992;86:968-978.

79 Tavi P, Han C, Weckstrom M: Mechanisms of stretch-induced changes in $\mathrm{Ca} 2+$ in rat atrial myocytes. Role of increased troponin $\mathrm{C}$ affinity and stretch-activated ion channels. Circ Res 1998;83:1165-1177.

80 Boudoulas H, Reynolds JC, Mazzaferri E, et al: Metabolic studies in mitral valve prolapse syndrome. A neuroendocrine-cardiovascular process. Circulation 1980;61:1200-1205.

-81 Boudoulas H, Reynolds JC, Mazzaferri E, et al: Mitral valve prolapse syndrome: the effect of adrenergic stimulation. J Am Coll Cardiol 1983;2:638-644.

82 Davies AO, Su CJ, Balasubramanyam A, et al: Abnormal guanine nucleotide regulatory protein in MVP dysautonomia: evidence from reconstitution of Gs. J Clin Endocrinol Metab 1991;72:867-875.

83 Anwar A, Kohn SR, Dunn JF, et al: Altered $\beta$-adrenergic receptor function in subjects with symptomatic mitral valve prolapse. Am J Med Sci 1991;302:9-17.

-84 Edwards JE: Mitral insufficiency resulting from 'overshadowing' of leaflets. Circulation 1971;43:606-612. 